\title{
Development of the Rehabilitation Health Policy, Systems, and Services Research Field: Quantitative Analyses of Publications over Time (1990-2017) and across Country Type
}

\author{
Tiago S. Jesus $1, * \mathbb{D}$, Helen Hoenig ${ }^{2,3}$ and Michel D. Landry ${ }^{4,5}$ \\ 1 Global Health and Tropical Medicine \& WHO Collaborating Center on Health Workforce Policy and Planning, \\ Institute of Hygiene and Tropical Medicine-NOVA University of Lisbon, 1349-008 Lisbon, Portugal \\ 2 Physical Medicine and Rehabilitation Service, Durham Veterans Administration Medical Center, Durham, \\ NC 27705, USA; Helen.Hoenig@va.gov \\ 3 Division of Geriatrics, Department of Medicine, Duke University Medical Center, Durham, NC 27710, USA \\ 4 School of Medicine, Duke University, Durham, NC 27710, USA; mike.landry@duke.edu \\ 5 Duke Global Health Institute, Duke University, Durham, NC 27710, USA \\ * Correspondence: jesus-ts@outlook.com; Tel.: +35-191-741-0478
}

Received: 3 January 2020; Accepted: 2 February 2020; Published: 4 February 2020

check for updates

\begin{abstract}
Background: Health policy, systems and services research (HPSSR) is increasingly needed to enable better access to, and value of, rehabilitation services worldwide. We aim to quantify the growth of Rehabilitation HPSSR publications since 1990, compared to that of overall rehabilitation research and overall HPSSR. Methods: Quantitative, comparative analysis of publication trends using the PubMed database and its indexation system. Comprehensive search filters, based on Medical Subject Headings (MeSH), were built and calibrated to locate research articles with content on HPSSR and rehabilitation of physical impairments. Additional filters were used for locating research publications declaring funding support, publications in rehabilitation journals, and finally publications focused on high-income (HICs) or low- and middle-income countries (LMICs). The same approach was used for retrieving data on comparator fields-overall HPSSR and overall rehabilitation research. Linear regressions, with ANOVA, were used for analyzing yearly publication growths over the 28-year time frame. Results: Rehabilitation HPSSR publications in PubMed have grown significantly from 1990 to 2017 in the percentage of all rehabilitation research (from $11 \%$ to $18 \%$ ) and all HPSSR (from $2.8 \%$ to $3.9 \%$; both $p<0.001$ ). The rate of Rehabilitation HPSSR published in rehabilitation journals did not change significantly over time $(p=0.47)$. The rates of publications with declared funding support increased significantly, but such growth did not differ significantly from that of the comparator fields. Finally, LMICs accounted for $9.3 \%$ of the country-focused rehabilitation HPSSR since 1990, but this percentage value increased significantly $(p<0.001)$ from $6 \%$ in 1990 to $13 \%$ in 2017 . Conclusion: Rehabilitation HPSSR publications, i.e., those indexed in PubMed with related MeSH terms, have grown in both absolute and relative values. Rehabilitation HPSSR publications focused on LMICs also grew significantly since 1990, but still remained a tiny portion of the Rehabilitation HPSSR publications with country-specific MeSH terms.
\end{abstract}

Keywords: health policy; health services research; rehabilitation; PubMed; publications

\section{Introduction}

Rehabilitation services are increasingly needed around the globe, following the global ageing population and the increased rates of chronic conditions and disabilities [1,2]. However, health systems 
worldwide are often under-resourced and ill-prepared to efficiently meet the rehabilitation needs of the population [3-9]. Production, dissemination, and uptake of rehabilitation-focused health policy, systems, and services research (i.e., Rehabilitation HPSSR) are a means to strengthen health systems' capacity to deliver appropriate, high-value rehabilitation services, toward sustainably meeting the growing rehabilitation needs of the population [10-14].

Differing from clinical or basic research, health policy and systems research addresses the upstream aspects of health, organizations, and policies [15-17]. It does it so as an inter-disciplinary field that seeks to understand and find solutions as to how: (1) societies and stakeholders organize themselves to achieve collective health goals; (2) health systems respond and adapt to health policies, and (3) health policies can shape and are shaped by health systems and broader determinants of health $[15,17]$. Health policy and systems research address any, and often multiple, 'building blocks' of health systems (e.g., governance, workforce, financing), within a systems-thinking approach toward promoting the coverage, quality, efficiency and equity of health systems [15,18-21]. At the service-delivery level, and with increasing convergence with a broader health systems research $[16,19,22]$, health services research refers to a multidisciplinary field of investigation on how social factors, financing, organizations, technologies, innovation, and personal behaviors affect the access to healthcare, its quality, and its cost $[23,24]$. Taken together, HPSSR addresses aspects within or at the interface of health policy, of health system's organization, and of healthcare delivery - for improved healthcare access, healthcare value, and population health.

In the rehabilitation field, advancing HPSSR is an increasing priority. This has been evident through recent publications in scientific journals on the role for and value of advancing rehabilitation health-services research [10,11,25-27], a number of empirical works, research syntheses and frameworks on rehabilitation health policy and health systems issues [12,28-30], rehabilitation stakeholders or interest groups with a focus on advancing rehabilitation services and services research [31,32], funding mechanisms or funding policies welcoming HPSSR in the rehabilitation field [33,34], and the recent input from the World Health Organization for developing a rehabilitation health policy and health systems research agenda [13].

Despite this growing interest, to our knowledge there is no systematic, quantitative analysis of the evolving research production on Rehabilitation HPSSR topics, even though its exists for other rehabilitation research areas [35-39] and for aspects of the overall HPSSR [21,40-45].

Hence, we aim to quantify the growth in the number of Rehabilitation HPSSR publications from 1990 and 2017, compared to that of overall rehabilitation research and overall HPSSR. This is operationalized through the following study questions:

1. What is the total amount, absolute growth, and relative growth of Rehabilitation HPSSR publications (i.e., as the percentage of all rehabilitation research publications, and as the percentage of all HPSSR publications)?

2. Which portion of the Rehabilitation HPSSR has been published in rehabilitation journals, and has that changed over time?

3. Which portion of the country-specific rehabilitation HPSSR publications focus on high-income countries (HICs) versus low- and middle-income countries (LMICs)?

4. What are the yearly rates of the Rehabilitation HPSSR publications that declare funding support, and how does that compare to rehabilitation research overall and HPSSR overall.

Answering these questions will provide information on crude trends in HPSSR articles in the rehabilitation literature from 1990 to 2017.

\section{Materials and Methods}

\subsection{Design}

Quantitative, comparative analysis of publication trends over time using data from the indexation system of a large health research database. 


\subsection{Data Source}

The PubMed database (http://www.ncbi.nlm.nih.gov/pubmed) and the indexation system for its largest sub-component (i.e., MEDLINE) was used for this analysis. Although not exhaustive, PubMed is a comprehensive research database. For instance, there is evidence that PubMed contains about $85 \%$ of the studies included in health-based systematic reviews $[46,47]$. Similarly, in a sample of randomized controlled trials relevant to physical therapy, PubMed indexed $89 \%$ of them, slightly more than EMBASE (88\%) and close to PEDro (92\%) and CENTRAL (95\%). Of note, PEDro and CENTRAL are databases specialized in physical therapy and clinical trials, respectively. PubMed has other advantages for this study-it is freely-accessible, hence the study is easily reproducible, and it addresses the health field as a whole, enabling comparative analyses.

PubMed/MEDLINE is suited for analyzing publication trends due its comprehensive indexation system, based on Medical Subject Headings (MeSH) that are organized within a hierarchical tree and assigned to each paper by trained indexers. Articles are therefore systematically indexed by research topic and methodology regardless specific words authors have used. Using such capabilities, PubMed has been a source for large analyses of publication trends within [35-37,39,48] and outside [49-51] the rehabilitation field. Here, we also relied fully on the PubMed indexing facilities (i.e., do not manually screen or review titles, abstracts or full texts) to identify rehabilitation HPSSR publications and those of comparator fields: overall HPSSR and overall rehabilitation research.

\subsection{Search Filters-Construction}

We used a mixed-methods approach for a comprehensive determination of search filters, i.e., combinations of relevant MeSH terms, for each component of our searches (e.g., HPSSR, rehabilitation, journal and country types).

\subsubsection{HPSSR}

For building the HPSSR filter, we first relied on working definitions and conceptual papers within the HPSSR arena [13,15-20,23,24]. Then, the key concepts extracted were translated into MeSH terms, using target and snowballing searches in the MeSH database [52]. For example, like other research [21], we searched the MeSH database for key terms around the six 'building blocks' of health systems (i.e., governance/leadership, workforce, financing, information systems, etc.). Additionally, we searched the MeSH database for other related key concepts (e.g., health planning, services supply and demand, health equity, quality improvement, healthcare access, etc.). The non-redundant terms (i.e., all of those not fully contained within upper-level MeSH terms indeed included) were combined as alternatives to one another (i.e., using the Bolean operator "OR"). We used the tag [majr] for each term, to retrieve only articles with any of those MeSH terms indexed as a Major Topic. This means that we selected only the research focused on a HPSSR topic, not those merely related to it.

\subsubsection{Rehabilitation}

We focused on the rehabilitation of people with physical impairments. Hence, some forms of rehabilitation were excluded, such as the rehabilitation of mental health conditions (psychiatric rehabilitation), substance abuse, people with sensory impairments per se (e.g., correction of hearing impairment), and people with oral health conditions. However, for example, the rehabilitation of cognitive, communicative, and neuro-behavioral impairments as a result of or associated to physical impairments were included in the scope of rehabilitation covered $[1,37,53]$. With that field restriction, and based on existing conceptual works or definitions of rehabilitation [1,53-57] as well as published search filters [35-37], we also approached the MeSH database to identify rehabilitation-related MeSH terms and any to be excluded with the Bolean operator "NOT"; for example, "psychiatric rehabilitation" $[\mathrm{MeSH}]$ is within the upper-level "rehabilitation" $[\mathrm{MeSH}]$, hence needed to be actively excluded. 
As above, we only included non-redundant MeSH terms, selected as a Major Topic. The exclusions were made with regular MeSH terms.

\subsubsection{Research Publications in Humans}

PubMed contains many publication types (e.g., editorial, letters, commentaries) that would not be eligible as research publications. Hence, based on previous search filters [35-37] and target searches in the MeSH database, we constructed a search filter for locating all kinds of empirical-based research publications, including qualitative research, case reports, systematic reviews, or practice guidelines. As systematic reviews have only been indexed as such in PubMed since 2019, we used a combined set of search terms to locate them, including non-MeSH terms [36,37] Finally, we narrowed down the research publications for those on "humans" subjects (e.g., not on animals). This filter applied to all the searches, including those targeting comparator fields.

\subsubsection{Journal Type}

We used the National Library of Medicine's journal catalog to identify journals that were indexed in PubMed and fit the scope of "physical medicine and rehabilitation" [sb], which includes, for example, physical and occupational therapy. As a result of the search, 116 journals were identified and the respective $\mathrm{MeSH}$ terms were retrieved as alternative to one another.

\subsubsection{Funding Support}

We use "Support of Research" [Publication Type] as the overarching MeSH term for articles declaring funding support from any venue.

\subsubsection{Country-Type}

We used the World Bank's classification to determine which countries and territories (e.g., Hong Kong) are high- or low-income, then translated these to the respective MeSH terms, if existent (i.e., not for a minority of small territories). These terms were set as alternative to one another (using the operator "OR") for the groups of HICs and LMICs. Of note, not all articles addressed specific countries (e.g., many systematic reviews), and articles could address no particular countries or HICs and LMICs at the same time. Also, when specific countries were addressed but this was not be explicit in the articles' title or abstract, often the articles were not granted a country-specific MeSH term. Hence, the amount of country-specific rehabilitation HPSSR is not necessarily equal to that of overall rehabilitation HPSSR.

\subsection{Search Filters-Calibration}

For calibrating the search filters, we ran pilot searches with or without some of the MeSH terms the research team appraised as at the borderline for inclusion or exclusion, assessing the impact they had on the search results. For example, we tested the collective impact of introducing a set of MeSH terms focused on assistive devices ("Self-Help Devices"[Majr] OR "Exoskeleton Device"[Majr] OR “Artificial Limbs"[Majr] OR “Orthotic Devices"[Majr] OR “Canes”[Majr] OR “Walkers"[Majr] OR "Crutches"[Majr]) within the search filter for rehabilitation. Including this whole set of terms added less than $2 \%$ more Rehabilitation HPSSR articles retrieved. Hence, the inclusion of this set was not deemed to carry substantial risk to preciseness (e.g., inclusion of too many articles not directly related to the rehabilitation: issues on device manufacturing, etc.), with likely benefits for increased sensitivity (e.g., identifying additional articles on the training or prescription of assistive devices). Theoretically, HPSSR cares about making medical products (including assistive technologies) available to those who need them [58]. Therefore, we retained this set of search terms in our final search strategy.

A final calibration exercise entailed a random selection of 30 articles from the authors' personal resources, published before 2017, that were deemed relevant for the field of Rehabilitation HPSSR by 
the research team. These articles were searched for in PubMed, and their major MeSH term retrieved. This whole process enabled the identification of one more relevant MeSH term for the rehabilitation search filter (i.e., "recovery of function" [MeSH]) and one more relevant term for the filter on research publications. For instance, some research articles (e.g., using mixed methods) were not indexed for any method/publication type in particular, but were indexed for "research support" [publication type]. Hence, we added this term to the research publications filter, assuming that all articles that declare "research support" are indeed research. This resulted in a substantial increase in the number of detected publications, sometimes over one-third more, with the added sensitivity coming with low risk to preciseness.

Appendix A provides the full set of calibrated search filters that we finally used, and the details of how these were combined in PubMed searches to answering our study questions.

\subsection{Searches Conduct and Time Span}

The searches were conducted in late October 2019 but were narrowed down in publication dates from January 1, 1990 to December 31, 2017. This accounted for the fact that "publication types" have been systematically indexed in PubMed only since 1990 and for the typical 2-year delay in the PubMed indexation.

Specifically, for the searches focused on funding support, the time span was narrowed down to end at December 2015. Indeed, in preliminary data visualizations, we observed outlier results (substantially lower number of publications for the years 2016 and 2017) in every search, across the fields, i.e., focused or not on rehabilitation or HPSSR. This suggests a delay or relevant change in this indexation process in particular, rather than reflecting changes in publications data itself. Hence, data on 2016 and 2017 for declared funding support were excluded.

\subsection{Data Extraction, Management and Analysis}

From each search in PubMed, the total and yearly volume of publications were exported to Excel (Microsoft Corporation). There, we computed relevant percentages (e.g., percentage of Rehabilitation HPSSR publications among all rehabilitation research publications, overall and for each year). As this paper involved comparative analyses, the data were often analyzed and reported in those relative percentages. Run charts and then linear, exponential or logarithmic regression models, according to best fit (determined by $r^{2}$ values and visual examination) were used to analyze any changes in the count data and computed percentages over time. We retained the linear models whenever the fit was similar (e.g., $r^{2}$ values difference $<0.02$ ).

We used simple linear regressions, with ANOVA, for determining whether any yearly changes, in counts or percent values, were significant. We use the JASP 0.10 .20 software (University of Amsterdam, Amsterdam, The Netherlands) for this analysis. $p$ values $<0.05$ were considered statistically significant.

\section{Results}

Below we present the results according to our initial research questions.

\subsection{Absolute and Relative Growth}

From 1990 to 2017, a total of 23,105 Rehabilitation HPSSR publications were found, with a significant linear growth of 63.3 publications a year (95\% Confidence Interval (CI): 58.2-68.4; $p<0.001 ; r^{2}=0.96$ ).

For the relative growth, Figure 1 shows that HPSSR publications accounted for $17.6 \%$ of the rehabilitation research publications in 2017, compared to $10.6 \%$ back in 1990 . This equates to a significant yearly growth $(p<0.001)$, albeit logarithmic in type $\left(r^{2}(\log\right.$ model $\left.)=0.83\right)$, i.e., more pronounced in the early years. 


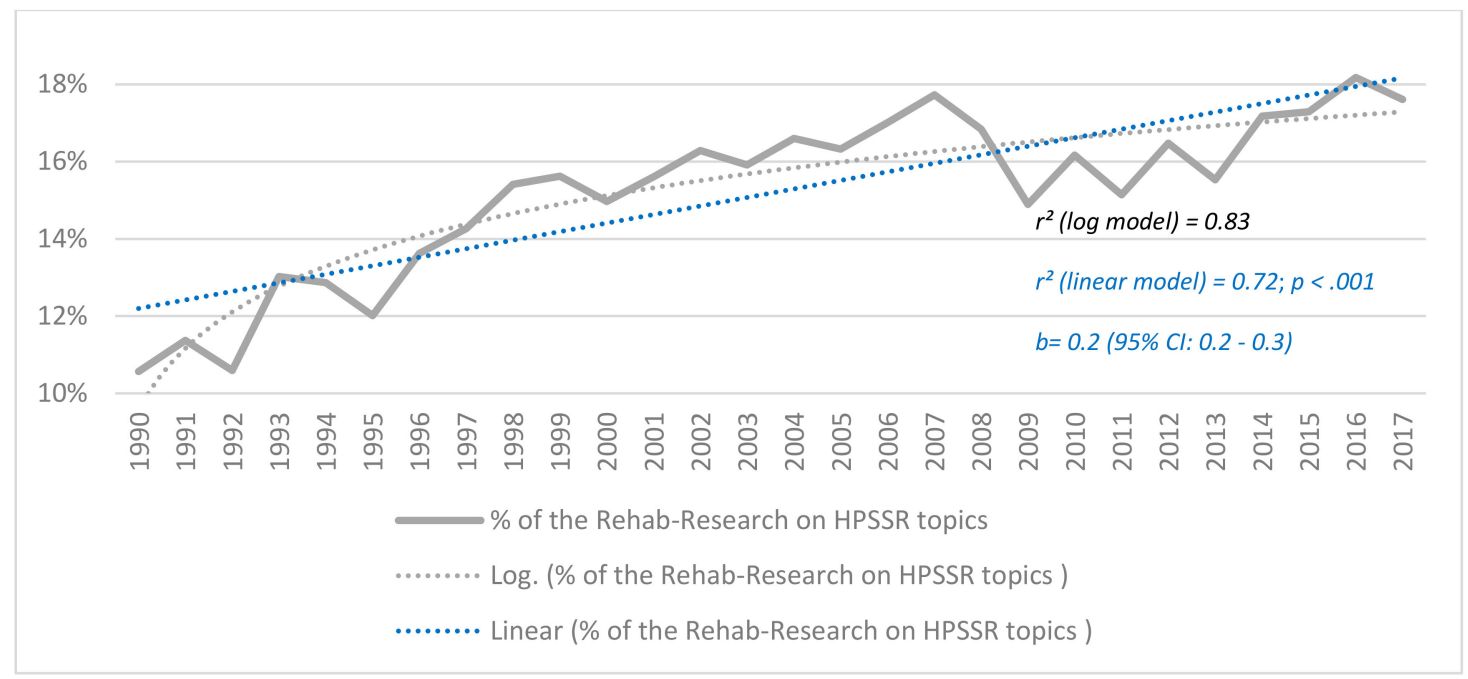

Figure 1. The yearly percentage of the Rehab (i.e., rehabilitation) research that is focused on Health Policy, Systems and Service Research (HPSSR) topics from 1990 to 2017, and the regression model (log: logarithmic) that had the best fit with the data. The $p$ value and $95 \%$ Confidence Interval (CI) refer to the linear regression model.

Figure 2, in turn, shows that rehabilitation accounted for 3.9\% of all HPSSR publications in 2017, compared to only $2.8 \%$ in 1990 , referring to a significant and linear growth in this relative value $\left(p<0.001 ; r^{2}\right.$ (linear model) $\left.=0.9\right)$.

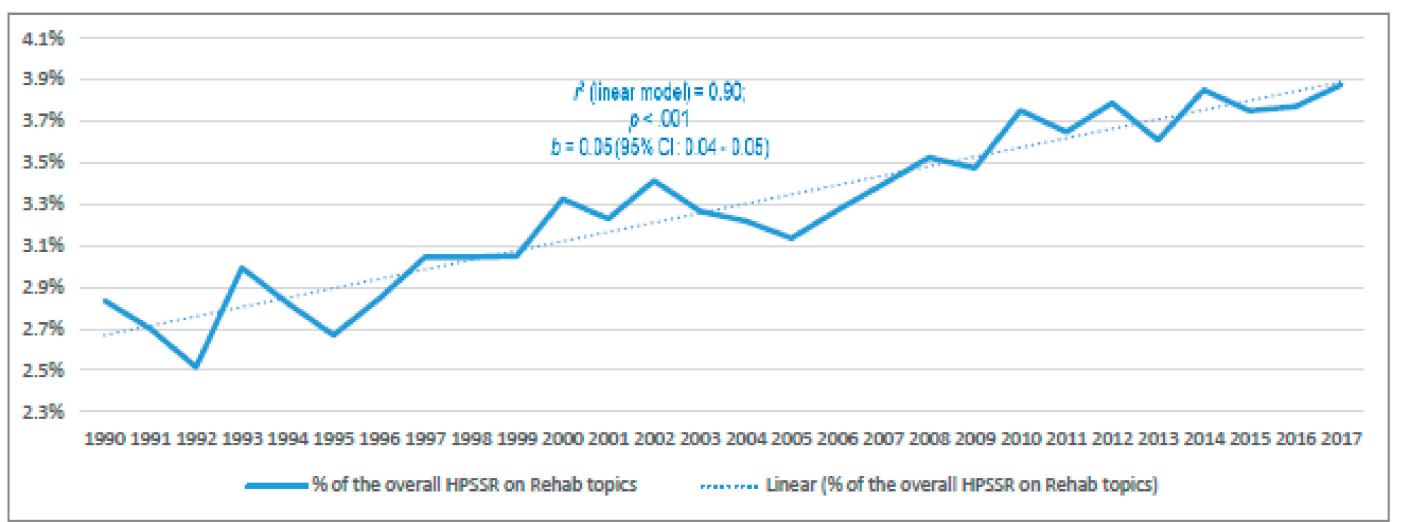

Figure 2. The yearly percentage of the Health Policy, Systems and Service Research (HPSSR) that is focused on Rehab (i.e., rehabilitation) topics from 1990 to 2017, and the regression model (linear) that had the best fit with the data. The $p$ value and 95\% Confidence Interval (CI) refer to the linear regression model.

\subsection{Journal Type}

Figure 3 shows that the percentage of Rehabilitation HPSSR published in rehabilitation journals did not significantly change over time $(p=0.49)$, erratically moving around $24 \%$. On the other hand, the percentage of the overall rehabilitation research (i.e., not only the Rehabilitation HPSSR) that was published in rehabilitation journals increased significant yet logarithmically over time $(p<0.001$; $r^{2}(\log$ model $\left.)=0.55\right)$, from $17 \%$ in 1990 to approaching $22 \%$ in 2017 , the typical values of the Rehabilitation HPSSR. 


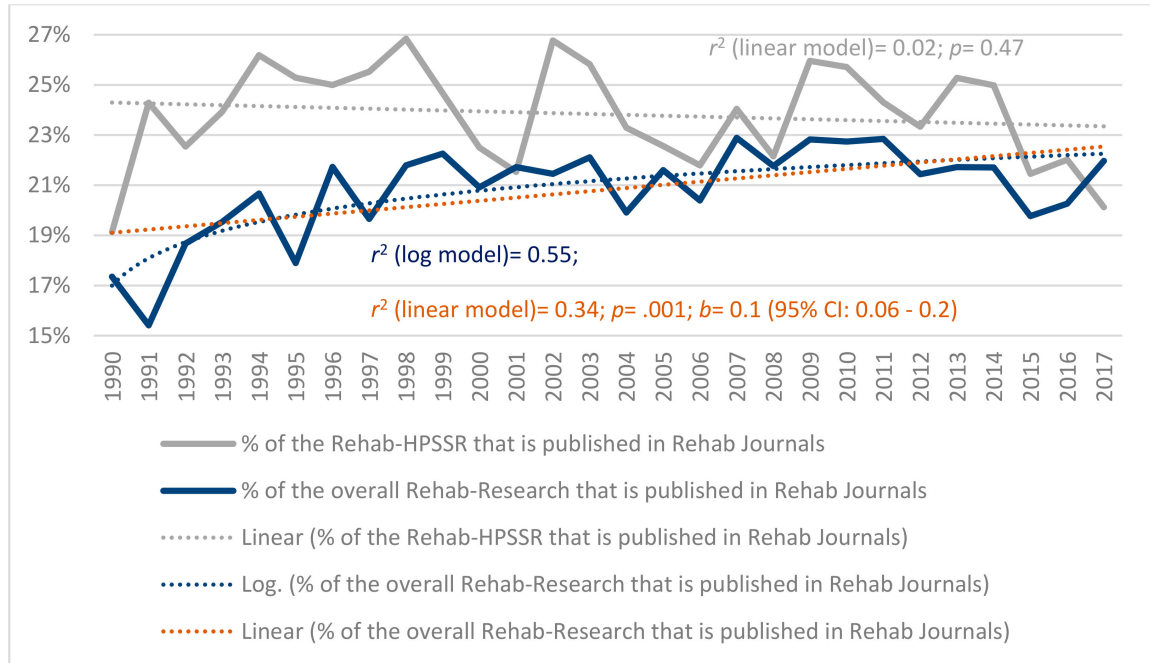

Figure 3. The yearly percentage of the rehab (i.e., rehabilitation) Health Policy, Systems and Service Research (HPSSR) and of the overall rehab research that were published in rehab journals from 1990 to 2017, and the regression models that had the best fit with the data. The $p$ values and $95 \%$ Confidence Interval (CI) refer to the linear regression models.

\subsection{HICs Versus LMICs}

From 1990 to 2017, a total of 9733 Rehabilitation HPSSR publications were indexed in PubMed with a focus on any HIC, and 986 on any LMIC. This means that LMICs account for up to $9.3 \%$ of the Rehabilitation HPSSR publications that had country-specific MeSH terms; however, Figure 4 shows that such a percentage value has been growing significantly over time $(p<0.001)$, from $6 \%$ in 1990 to $13 \%$ in 2017.

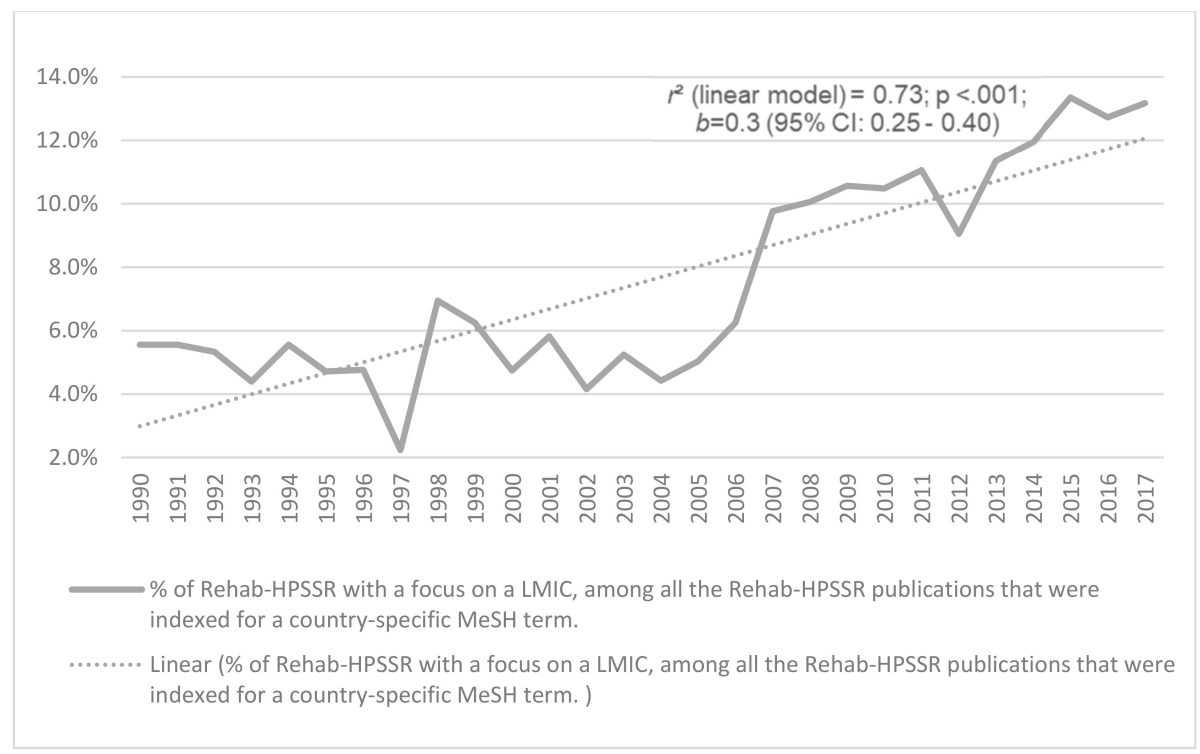

Figure 4. The yearly percentage of the rehab (i.e., rehabilitation) Health Policy, Systems and Service Research (HPSSR) focused on Low- and Middle-Income Income Countries (LMICs) among all rehab-HPSSR publications with a country-specific Medical Subject Heading [1990-2017], and the regression model (i.e. the linear) that had the best fit with the data. The $p$ value and $95 \%$ Confidence Interval (CI) refer to the linear regression model. 


\subsection{Funding Support Declared}

From 1990 to 2015, 11,037 Rehabilitation HPSSR publications declared funding support, i.e., 47.8\% of them. Figure 5 shows that the proportion of publications declaring support increased significantly over time, linearly from $43 \%$ (1990) to 58\% (2015) ( $p<0.001 ; 95 \%$ CI: 58.2-68.4; $\left.r^{2}=0.96\right)$. This growth was comparable to (i.e., $95 \%$ CIs partly overlapped) rehabilitation research as a whole and HPSSR as a whole, with all three approaching 2015 with values within the $53-58 \%$ range.

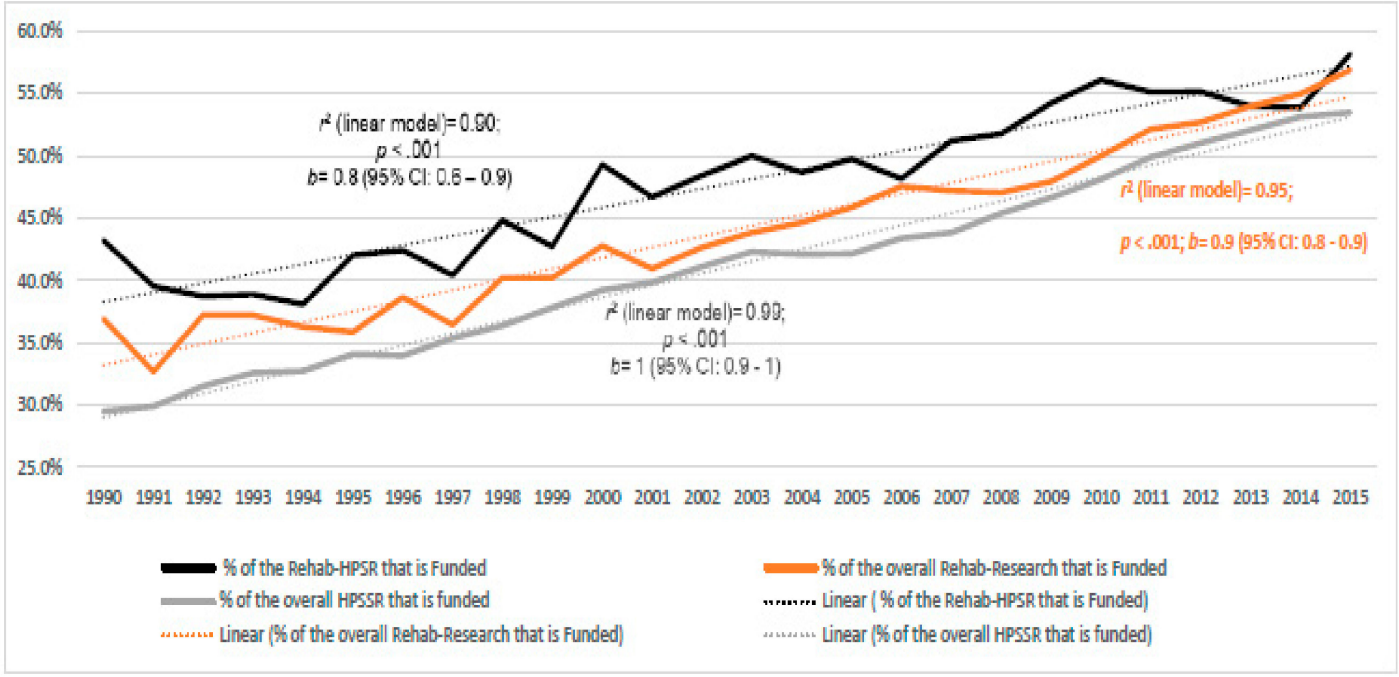

Figure 5. The yearly percentage of the research publications that were funded for the rehab (i.e., rehabilitation) Health Policy, Systems and Service Research (HPSSR), the overall rehab research, and the overall HPSSR (1990-2015), and the regression models that had the best fit with the data. The $p$ values and $95 \%$ Confidence Intervals (CIs) refer to the linear regression model.

\section{Discussion}

Rehabilitation HPSSR publications, notably those indexed for rehabilitation and HPSSR topics in the PubMed database, have growth significantly from 1910 to 2017, in both absolute and relative values. Indeed, the growth of the rehabilitation HPSSR publications significantly outpaced that of both comparators, i.e., grew significantly in the percentage of all rehabilitation research, and in the percentage of all HPSSR. We also observed discrepancies in the Rehabilitation HPSSR publications focused on HICs versus LMICs, with the latter accounting for $9.3 \%$ of the Rehabilitation HPSSR publications that had at least a country-specific MeSH term. However, a significant, growing trend from $6 \%$ to $13 \%$ (1990-2017) was observed in that percentage value. Below we present five points of discussion related to our findings.

First, the observed growth of publications on HPSSR topics within the overall rehabilitation research, i.e., from $11 \%$ to $18 \%$ (1990-2017), means that rehabilitation stakeholders are conducting, comparatively, more HPSSR. Nonetheless, HPSSR topics still represent less than one-fifth of all rehabilitation research publications and the observed relative growth was less pronounced (i.e., flattening) in more recent years. It is hard to know what is the desirable figure with regard to the proportion of rehabilitation research that should be devoted to HPSSR; however, what we do know is that Rehabilitation HPSSR helps to enhance the value or population impact of other forms of rehabilitation research (e.g., clinical rehabilitation research). For example, HPSSR would help to translate the growing clinical knowledge into more common, more accessible, and more affordable rehabilitation practices, boost innovation and accountability for service organization and delivery, and make generalizable knowledge responsive or adaptive to identified local needs $[10,11,15,59,60]$.

Second, Rehabilitation HPSSR represents a significantly increased proportion of publications within HPSSR, from $2.8 \%$ in 1990 to $3.9 \%$ in 2017 . The growth over time has been significant 
and relatively linear, i.e., constant, over the 28 -year period; nonetheless, rehabilitation represents a very small portion of all HPSSR. This may be concerning given the current high levels of physical rehabilitation needs worldwide (e.g., in 2017, 40\% of the world's non-fatal health loss came from conditions amenable to physical rehabilitation [1]), the significant growth in those needs observed across country and condition types [1,61], the evidence for large gaps in the physical rehabilitation supply [6,29], and the growing evidence on the individual, economic, and broader societal benefits associated to the delivery of appropriate physical rehabilitation services [2,62-65]. In other words, we have found that the amount of Rehabilitation HPSSR is a disproportionate to the global population's needs for such services. All accounted for, we suggest that there is a urgent need to address the collective negligence and limited development of rehabilitation resources within and across many countries $[2,6,8,9]$, the gaps in quality, access, and value of rehabilitation care worldwide $[5,6,8,9,14]$, and the growing unmet needs for rehabilitation across countries of all income levels $[1,6]$.

Third, LMICs accounted for a relatively marginal share of the country-specific Rehabilitation HPSSR publications, i.e., less than $10 \%$ for the all 28 years analyzed. This diverges, for example, from the overall health policy and systems research, which is close to a $50-50 \%$ distribution between HICs and LMICs [21], with publications relevant to LMICs being those increasing at the highest rates [40]. The seminal World Report on Disability [66] estimated that $80 \%$ of people with disabilities live in LMICs, a recent analyses of data from the Global Burden of Disease 2017 found that LMICs account for $77 \%$ of the physical rehabilitation needs [1], while several reports point towards large unmet rehabilitation needs in LMICs $[5,6,8,9]$. While we found a significant growing trend in the portion of the country-specific Rehabilitation HPSSR publications that were focused on LMICs(up to $13 \%$ in 2017), a still higher preponderance of rehabilitation HPSSR is likely needed, especially in LMICs; transformational rather than incremental improvements would be required to address this gap. Capacity would need to be created, for example, in developing a research workforce with the means, focus, and capabilities to implement a Rehabilitation HPSSR agenda worldwide [10,22,67-69], including with a special focus on LMICs [70-72].

Fourth, we did not observe a significant change in the rate of Rehabilitation HPSSR publications in rehabilitation journals. Given that we found that less than $25 \%$ of rehabilitation HPSSR publications are in rehabilitation journals and given that we lack databases or repositories specific to Rehabilitation HPSSR, persons wanting to locate and use Rehabilitation HPSSR evidence will need to rely on comprehensive search strategies in large health databases.

Finally, we found a significant increase in the rates of Rehabilitation HPSSR declaring funding support and those rates are comparable to similar research. This may be comforting for the rehabilitation research community; however, this finding occurs against the backdrop of overall stagnation or reduction in funding for health services research in the US [45], with US health services research representing a mere $0.3 \%$ of the total US healthcare expenditures or only $5 \%$ of all research funding [73]. While in low-income countries the funding availability for the overall health policy and systems research has increased, notably to institutions in Sub-Saharan Africa [21], we found the number of publications focused on LMICs was insufficient to enable an analysis of the funding trends over time on the Rehabilitation HPSSR focusing on LMICs.

\section{Limitations}

This paper has several limitations:

We included only research publications indexed in PubMed, not the full spectrum of research publications. Similarly, we fully relied on the PubMed indexation system for this large dataset analysis, while the indexation system is not fully sensitive and precise. Nonetheless, the PubMed indexation refers to a standard process conducted by trained indexers. Also, the same search terms, comprehensively developed, were applied across the comparator fields. As the results are essentially reported in relative percentages, this makes the comparative results less prone to bias. Furthermore, due to typical delays in the PubMed indexation, we could only present results up to 2017 (and those for funding only to 
2015); hence, the effects of more recent pushes for advancing the Rehabilitation HPSSR [10,11,13,34] may not be noticed yet. It is also worth noting that we analyzed merely the number of research publications, not their methodological type or quality. Besides, we were unable to analyze the yearly evolution of the Rehabilitation HPSSR in LMICs that have had declared funding support, given the low number of publications per year. Furthermore, we did not analyze the country of origin of the authors or the authors' network [42]. Additionally, only about half of the Rehabilitation HPSSR publications in PubMed had a country-specific MeSH term (e.g., were systematic reviews or did not explicitly address a country in the article's title or abstract); hence the analysis by country-type was carried out over a sample of the Rehabilitation HPSSR publications in PubMed, although this does not preclude a biased sample, i.e., not necessarily favoring HICs or LMICs.

Moreover, we did not analyze the specific scope of Rehabilitation HPSSR by thematic areas [21,44], but solely the whole of these research publications. Similarly, we did not analyze publications from the field of health policy and systems research separately from those in the field of health services research, as both broadly aim to address issues of healthcare access, quality, and value at the population level $[16,19,22]$, thereby it would be complex to select MeSH terms clearly relevant for one but clearly not relevant for the other. Furthermore, we analyzed rates of funding support that research publications declared, not funding support amounts [45].

Finally, we focused only on research publications, while perspectives, commentaries, conceptual, narrative or other types of article, e.g., analyzing current situations [74] or describing development or consultation activities [75], have an important role as well.

Highlighting these limitations are critical in pinpointing the weaknesses of our methodology; however, given the limited availability of the publicly available information, we interpret this study as a starting point, and would invite the rehabilitation and/or HPSSR community to contribute to this discussion.

\section{Conclusions}

This research uses the PubMed indexation capabilities to provide information on crude trends (i.e., publications data not manually screened) on the evolution of the number of Rehabilitation HPSSR articles published from 1990 to 2017. With such a strategy, we have found that Rehabilitation HPSSR publications, i.e., those published and indexed in PubMed with rehabilitation- and HPSSR-related MeSH terms, have grown in both absolute and relative values. However, in 2017, Rehabilitation HPSSR still accounted for merely $18 \%$ of the rehabilitation research publications and $4 \%$ of the overall HPSSR publications in PubMed. We also found that the portion of Rehabilitation HPSSR publications focused on LMICs, among those that had country-specific MeSH terms, has been growing significantly since 1990, but is still minute, especially given the sizeable unmet needs for rehabilitation services in many LMICs.

Author Contributions: Conceptualization, T.S.J., H.H., and M.D.L.; Methodology, T.S.J.; Data Curation, T.S.J.; Writing—Original Draft Preparation, T.S.J.; Writing—Review \& Editing, T.S.J., H.H., and M.D.L.; Visualization, T.S.J.; Formal Analysis, T.S.J.; Project Administration, T.S.J.; Supervision, M.D.L., and H.H. All authors have read and agreed to the published version of the manuscript.

Funding: This research received no external funding.

Conflicts of Interest: The authors declare no conflicts of interest.

\section{Appendix A Search Strategy}

\section{Introductory Notes}

- In this Appendix, we present the complete search filters that were used. They enable the reproduction of the searches in PubMed (https://www.ncbi.nlm.nih.gov/pubmed). Of note, as the indexation process in PubMed is an ongoing endeavor; hence, values retrieved can be slightly 
higher than those presented in the results, especially for the number of articles published in the more recent years.

- Whenever reproducing the target searches, one needs to combine the relevant search filters below, using the Bolean operator "AND" in between.

For example, in the search aimed at locating rehabilitation HPSSR, the following combination of search filters must be employed: the Common Filter (used into all searches) AND the HPSSR filter AND the Rehabilitation Filter.

- Search filters were based essentially on MeSH terms. All lower-level MeSH Terms, i.e., all under those actually written, are included by default, unless otherwise specified (i.e., with the use of the operator "NOT").

- The hierarchical tree of MeSH terms, their definitions, and which MeSH terms are included under each MeSH term can be consulted at: https://www.ncbi.nlm.nih.gov/mesh.

\section{Search filters}

Common Filter (Used into all the Searches)—Research Publications, Humans, and Date Limits

("Study Characteristics" [Publication Type] OR "Support of Research" [Publication Type] OR "Guideline" [Publication Type] OR "Empirical Research"[MeSH] OR "Epidemiologic Methods"[MeSH] OR (Review[ptyp] AND systematic[tw] AND systematic[sb]) OR "Cochrane Database Syst Rev"[Journal] OR ("systematic review"[ti] OR "scoping review"[ti] OR "realist review"[ti])) AND (“humans"[MeSH Terms]) AND (“1990/01/01”[PDAT]: “2017/12/31”[PDAT])

\section{HPSR Filter}

(“Health Policy”[Majr] OR “Health Planning”[Majr] OR “Health Services Administration”[Majr] OR "Delivery of Health Care"[Majr] OR "Professional Competence"[Majr] OR "Clinical Competence"[Majr] OR "Race Factors"[Majr] OR "Advance Directive Adherence"[Majr] OR "Guideline Adherence"[Majr] OR "Independent Medical Evaluation"[Majr] OR "Organizational Case Studies"[Majr] OR "Outcome and Process Assessment (Health Care)"[Majr] OR "Patient Satisfaction"[Majr] OR "Program Evaluation"[Majr] OR "Root Cause Analysis"[Majr] OR "Peer Review, Health Care"[Majr] OR "Standard of Care"[Majr] OR “Inservice Training"[Majr] OR "Education, Continuing"[Majr] OR "Health Services Research"[Majr] OR “Healthcare Financing"[Majr] OR "Health Information Systems"[Majr] OR "Health Information Management"[Majr] OR “Health Information Interoperability"[Majr] OR "Information Technology"[Majr] OR “Organizational Innovation"[Majr] OR "Health Care Reform"[Majr] OR "Knowledge Management"[Majr] OR "Diffusion of Innovation"[Majr] OR “Change Management”[Majr])

Note: Even though not written down, relevant MeSH terms such as but not limited to "Health Services Needs and Demand"[Majr] OR "Health Workforce"[Majr] OR "Health Services Accessibility"[Majr] OR "Health Equity" [Majr] are included into the search filter (i.e., fully contained within upper-level MeSH terms included, such as but not limited to "Health Administration"[Majr]). In practice, adding any of the terms exemplified above to the search filter do not change the total number of articles retrieved.

\section{Rehabilitation Filter}

("rehabilitation"[Subheading] OR "Rehabilitation"[Majr] OR "Recovery of Function"[Majr] OR "Physical Therapy Specialty"[Majr] OR "Physical Therapy Modalities"[Majr] OR "Physical Therapy Department, Hospital"[Majr] OR "Hospitals, Rehabilitation"[Majr] OR "Physical Therapist Assistants"[Majr] OR "Physical Therapists"[Majr] OR "Physical and Rehabilitation Medicine"[Majr] OR "Rehabilitation Nursing"[Majr] OR "Occupational Therapists"[Majr] OR "Occupational Therapy Department, Hospital"[Majr] OR “Occupational Therapy"[Majr] OR "Speech-Language 
Pathology"[Majr] OR “Activities of Daily Living"[Majr] OR "Self-Help Devices"[Majr] OR "Exoskeleton Device"[Majr] OR “Artificial Limbs"[Majr] OR “Orthotic Devices"[Majr] OR "Canes"[Majr] OR "Walkers"[Majr] OR "Crutches"[Majr] OR "Rehabilitation Centers"[Majr] OR "Rehabilitation Research"[Majr] NOT "Correction of Hearing Impairment"[Mesh] NOT "Substance Abuse Treatment Centers"[Mesh] NOT "Mouth Rehabilitation"[Mesh] NOT “Mental Disorders"[Mesh] NOT "United States Substance Abuse and Mental Health Services Administration"[Mesh] NOT "National Institute of Mental Health (U.S.)"[Mesh] NOT "Mental Health Services"[Mesh] NOT "Mental Health Associations"[Mesh] NOT "Community Mental Health Services"[Mesh] NOT “Community Mental Health Centers"[Mesh] NOT "Rehabilitation, Vocational"[Mesh] NOT "Sheltered Workshops"[Mesh] NOT "Psychiatric Nursing"[Mesh] NOT "Mental Health Recovery"[Mesh] NOT "Psychiatric Rehabilitation"[Mesh])

Note: Even though not written down, relevant MeSH terms such as but not limited to "Neurological Rehabilitation"[Majr] and "Cardiac Rehabilitation"[Majr] are included into the search filter (i.e., fully contained within upper-level MeSH terms included, such as "Rehabilitation"[Majr]). In practice, adding any of the illustrated MeSH terms to the search filter above do not change the total number of articles retrieved.

\section{Funding support Filter}

"Support of Research" [Publication Type]

Note: This filter contains all the forms of funding support.

\section{Journals-All Listed for Physical and Rehabilitation Medicine[st] in National Library of Medice Catalog}

("Z Physiother"[Journal] OR “Vopr Kurortol Fizioter Lech Fiz Kult"[Journal] OR “Trans Am Clin Climatol Assoc"[Journal] OR "Train Sch Bull (Vinel)"[Journal] OR “Torture"[Journal] OR “Top Stroke Rehabil"[Journal] OR "Spec Educ Forward Trends"[Journal] OR "Spec Educ"[Journal] OR "Soc Rehabil Rec"[Journal] OR "Scand J Rehabil Med Suppl"[Journal] OR "Scand J Rehabil Med"[Journal] OR "Scand J Occup Ther"[Journal] OR "Rheumatol Rehabil"[Journal] OR "Rheumatol Phys Med"[Journal] OR "Rev Bras Fisioter"[Journal] OR "Rehabilitation (Bonn)"[Journal] OR "Rehabil Rec"[Journal] OR "Rehabil Psychol"[Journal] OR "Rehabil Nurs"[Journal] OR "Rehabil Lit"[Journal] OR “Rehabilitation (Stuttg)"[Journal] OR “Rehabilitacion (Madr)"[Journal] OR “Rehabil Fyz Lek"[Journal] OR “Rehab Manag"[Journal] OR “Record (Washington)"[Journal] OR "Radiobiol Radioter Fis Med"[Journal] OR “Qual Life Res"[Journal] OR "Prog Phys Ther"[Journal] OR "Presse Therm Clim"[Journal] OR "PM R"[Journal] OR "Physiother Theory Pract"[Journal] OR "Physiother Res Int"[Journal] OR "Physiotherapy"[Journal] OR "Phys Ther Sport"[Journal] OR "Phys Ther"[Journal] OR "Phys Occup Ther Pediatr"[Journal] OR "Phys Med Rehabil Clin N Am"[Journal] OR “Pediatr Rehabil"[Journal] OR “Pediatr Phys Ther"[Journal] OR “OTJR (Thorofare N J)"[Journal] OR “Ortop Traumatol Rehabil”[Journal] OR “Orizz Ortop Odie Riabil”[Journal] OR “Occup Ther Int"[Journal] OR “Occup Ther Health Care"[Journal] OR "Neurorehabil Neural Repair"[Journal] OR "NeuroRehabilitation" [Journal] OR "Neuropsychol Rehabil"[Journal] OR "Musculoskelet Sci Pract"[Journal] OR “Man Ther" [Journal] OR "J Assoc Phys Ment Rehabil"[Journal] OR "J Am Paraplegia Soc"[Journal] OR "J Sport Rehabil"[Journal] OR “J Spinal Cord Med"[Journal] OR "J Soc Work Disabil Rehabil”[Journal] OR “J Rehabil Res Dev Clin Suppl”[Journal] OR “J Rehabil Res Dev"[Journal] OR “J Rehabil R D"[Journal] OR “J Rehabil Med Suppl"[Journal] OR “J Rehabil Med"[Journal] OR “J Rehabil"[Journal] OR “J Physiother"[Journal] OR “J Pediatr Rehabil Med"[Journal] OR “J Outcome Meas"[Journal] OR "J Orthop Sports Phys Ther"[Journal] OR "J Occup Rehabil"[Journal] OR "J Neurol Phys Ther"[Journal] OR "J Neuroeng Rehabil"[Journal] OR "J Man Manip Ther"[Journal] OR "J Head Trauma Rehabil"[Journal] OR “J Hand Ther"[Journal] OR “J Geriatr Phys Ther"[Journal] OR “J Dance 
Med Sci"[Journal] OR “J Cardiopulm Rehabil Prev"[Journal] OR "J Cardiopulm Rehabil"[Journal] OR “J Burn Care Rehabil”[Journal] OR “J Bodyw Mov Ther"[Journal] OR “J Back Musculoskelet Rehabil"[Journal] OR "J Belge Rhumatol Med Phys"[Journal] OR "J Belge Med Phys Rehabil"[Journal] OR “J Belge Med Phys"[Journal] OR “Int Rehabil Med"[Journal] OR “Int J Rehabil Res"[Journal] OR “Int Disabil Stud"[Journal] OR "IEEE Trans Rehabil Eng"[Journal] OR "IEEE Trans Neural Syst Rehabil Eng"[Journal] OR "IEEE Int Conf Rehabil Robot"[Journal] OR "Fysiatr Revmatol Vestn"[Journal] OR “Eur J Phys Rehabil Med"[Journal] OR “Eura Medicophys"[Journal] OR “Disabil Rehabil Assist Technol"[Journal] OR “Disabil Rehabil"[Journal] OR “Dev Neurorehabil"[Journal] OR “Clin Rehabil" [Journal] OR "Can J Occup Ther"[Journal] OR “Bull Prosthet Res"[Journal] OR "Braz J Phys Ther"[Journal] OR "Aust Occup Ther J"[Journal] OR “Assist Technol"[Journal] OR "Artif Limbs"[Journal] OR "ARN J"[Journal] OR "Arch Phys Med Rehabil"[Journal] OR "Arch Phys Ther (Leipz)"[Journal] OR “Annu Rev Rehabil"[Journal] OR “Ann Phys Med"[Journal] OR “Ann Phys Rehabil Med"[Journal] OR “Ann Readapt Med Phys"[Journal] OR “Am Rehabil"[Journal] OR "Am J Phys Med Rehabil"[Journal] OR “Am J Phys Med"[Journal] OR “Am J Occup Ther"[Journal] OR “Am Correct Ther J"[Journal] OR “Adv Clin Rehabil"[Journal] OR “Acta Belg Med Phys"[Journal])

\section{Country-types}

High-Income:

("Argentina"[Mesh] OR "Chile"[Mesh] OR "Uruguay"[Mesh] OR "Panama"[Mesh] OR "United Kingdom"[Mesh] OR "Switzerland"[Mesh] OR "Sweden"[Mesh] OR "Spain"[Mesh] OR "Slovenia"[Mesh] OR "Slovakia"[Mesh] OR "Sint Maarten"[Mesh] OR "Netherlands"[Mesh] OR "Seychelles"[Mesh] OR "San Marino"[Mesh] OR "Saint Kitts and Nevis"[Mesh] OR "Portugal"[Mesh] OR "Poland"[Mesh] OR "Palau"[Mesh] OR "Norway"[Mesh] OR "New Caledonia"[Mesh] OR “Monaco"[Mesh] OR "Malta"[Mesh] OR "Luxembourg"[Mesh] OR "Lithuania"[Mesh] OR “Liechtenstein"[Mesh] OR "Latvia"[Mesh] OR "Italy"[Mesh] OR "Ireland"[Mesh] OR "Hungary"[Mesh] OR "Guam"[Mesh] OR "Greenland"[Mesh] OR "Greece"[Mesh] OR "Gibraltar"[Mesh] OR “Germany"[Mesh] OR "Polynesia"[Mesh] OR “France" OR "Finland"[Mesh] OR “Denmark"[Mesh] OR “Estonia"[Mesh] OR "Czech Republic"[Mesh] OR “Cyprus"[Mesh] OR “Curacao"[Mesh] OR "Croatia"[Mesh] OR "Channel Islands"[Mesh] OR "British Virgin Islands"[Mesh] OR "Bermuda"[Mesh] OR "Barbados"[Mesh] OR "Trinidad and Tobago"[Mesh] OR "Bahamas"[Mesh] OR "Austria"[Mesh] OR "Aruba"[Mesh] OR "Antigua and Barbuda"[Mesh] OR "Andorra"[Mesh] OR "United Arab Emirates"[Mesh] OR "Qatar"[Mesh] OR "Saudi Arabia"[Mesh] OR “Israel"[Mesh] OR "Kuwait"[Mesh] OR "Bahrain"[Mesh] OR "Singapore"[Mesh] OR "Japan"[Mesh] OR “Taiwan"[Mesh] OR "Hong Kong"[Mesh] OR "Macau"[Mesh] OR "Brunei"[Mesh] OR "Republic of Korea"[Mesh] OR "Australia"[Mesh] OR “New Zealand"[Mesh] OR “Canada"[Mesh] OR “United States"[Mesh])

\section{LMICs:}

("Africa"[Mesh] OR ("China"[Mesh] NOT “Hong Kong"[Mesh] NOT "Macau"[Mesh]) OR "Democratic People's Republic of Korea"[Mesh] OR "Mongolia"[Mesh] OR "Asia, Central"[Mesh] OR "Asia, Northern"[Mesh] OR "Bangladesh"[Mesh] OR "Bhutan"[Mesh] OR "India"[Mesh] OR “Nepal”[Mesh] OR “Pakistan"[Mesh] OR "Sri Lanka”[Mesh] OR “Afghanistan"[Mesh] OR "Iran"[Mesh] OR "Iraq"[Mesh] OR "Jordan"[Mesh] OR "Lebanon"[Mesh] OR "Syria"[Mesh] OR “Turkey"[Mesh] OR "Yemen"[Mesh] OR "Cambodia"[Mesh] OR "Indonesia"[Mesh] OR "Laos"[Mesh] OR "Malaysia"[Mesh] OR "Myanmar"[Mesh] OR "Philippines"[Mesh] OR "Thailand"[Mesh] OR “Timor-Leste"[Mesh] OR "Vietnam"[Mesh] OR “Mexico"[Mesh] OR "Belize"[Mesh] OR "Costa Rica"[Mesh] OR "El Salvador"[Mesh] OR "Guatemala"[Mesh] OR "Honduras"[Mesh] OR "Nicaragua"[Mesh] OR "Cuba"[Mesh] OR "Dominica"[Mesh] OR "Dominican Republic"[Mesh] OR "Grenada"[Mesh] OR "Haiti"[Mesh] OR "Jamaica"[Mesh] OR "Saint Lucia"[Mesh] OR "Saint Vincent and the Grenadines"[Mesh] OR "Bolivia"[Mesh] OR "Brazil"[Mesh] OR “Colombia"[Mesh] OR "Ecuador"[Mesh] OR "Paraguay"[Mesh] OR 
"Peru"[Mesh] OR "Suriname"[Mesh] OR "Venezuela"[Mesh] OR "Albania"[Mesh] OR "Bosnia and Herzegovina"[Mesh] OR “Bulgaria"[Mesh] OR “Kosovo"[Mesh] OR “Macedonia (Republic)"[Mesh] OR "Montenegro"[Mesh] OR "Moldova"[Mesh] OR "Republic of Belarus"[Mesh] OR "Romania"[Mesh] OR "Serbia"[Mesh] OR "Ukraine"[Mesh] OR “Transcaucasia"[Mesh] OR "Kazakhstan"[Mesh] OR “Kyrgyzstan"[Mesh] OR "Uzbekistan"[Mesh] OR "Samoa"[Mesh] OR ("Micronesia"[Mesh] NOT “Guam"[Mesh] NOT "Palau”[Mesh]) OR “Fiji”[Mesh] OR “Papua New Guinea”[Mesh] OR "Vanuatu"[Mesh] OR "Tonga"[Mesh])

\section{References}

1. Jesus, T.S.; Landry, M.D.; Hoenig, H. Global Need for Physical Rehabilitation: Systematic Analysis from the Global Burden of Disease Study 2017. Int. J. Environ. Res. Public Health 2019, 16, 980. [CrossRef] [PubMed]

2. Krug, E.; Cieza, A. Strengthening health systems to provide rehabilitation services. Bull. World Health Organ. 2017, 95, 167. [CrossRef] [PubMed]

3. Foster, M.; Allen, S.; Fleming, J. Unmet health and rehabilitation needs of people with long-term neurological conditions in Queensland, Australia. Health Soc. Care Community 2015, 23, 292-303. [CrossRef] [PubMed]

4. Fuentes, M.M.; Wang, J.; Haarbauer-Krupa, J.; Yeates, K.O.; Durbin, D.; Zonfrillo, M.R.; Jaffe, K.M.; Temkin, N.; Tulsky, D.; Bertisch, H.; et al. Unmet Rehabilitation Needs after Hospitalization for Traumatic Brain Injury. Pediatrics 2018, 141, e20172859. [CrossRef]

5. Kamalakannan, S.; Venkata, M.G.; Prost, A.; Natarajan, S.; Pant, H.; Chitalurri, N.; Goenka, S.; Kuper, H. Rehabilitation Needs of Stroke Survivors after Discharge from Hospital in India. Arch. Phys. Med. Rehabil. 2016, 97, 1526-1532. [CrossRef]

6. Kamenov, K.; Mills, J.A.; Chatterji, S.; Cieza, A. Needs and unmet needs for rehabilitation services: A scoping review. Disabil. Rehabil. 2019, 41, 1227-1237. [CrossRef]

7. Singh, R.; Sinha, S.; Bill, A.; Turner-Stokes, L. Unmet need for specialised rehabilitation following neurosurgery: Can we maximise the potential cost-benefits? Br. J. Neurosurg. 2017, 31, 249-253. [CrossRef]

8. Bright, T.; Wallace, S.; Kuper, H. A Systematic Review of Access to Rehabilitation for People with Disabilities in Low- and Middle-Income Countries. Int. J. Environ. Res. Public Health 2018, 15, 2165. [CrossRef]

9. Magnusson, D.; Sweeney, F.; Landry, M. Provision of rehabilitation services for children with disabilities living in low- and middle-income countries: A scoping review. Disabil. Rehabil. 2019, 41, 861-868. [CrossRef]

10. Graham, J.E.; Middleton, A.; Roberts, P.; Mallinson, T.; Prvu-Bettger, J. Health Services Research in Rehabilitation and Disability-The Time is Now. Arch. Phys. Med. Rehabil. 2018, 99, 198-203. [CrossRef]

11. Rundell, S.D.; Goode, A.P.; Friedly, J.L.; Jarvik, J.G.; Sullivan, S.D.; Bresnahan, B.W. Role of Health Services Research in Producing High-Value Rehabilitation Care. Phys. Ther. 2015, 95, 1703-1711. [CrossRef] [PubMed]

12. McVeigh, J.; MacLachlan, M.; Gilmore, B.; McClean, C.; Eide, A.H.; Mannan, H.; Geiser, P.; Duttine, A.; Mji, G.; McAuliffe, E.; et al. Promoting good policy for leadership and governance of health-related rehabilitation: A realist synthesis. Glob. Health 2016, 12, 49. [CrossRef] [PubMed]

13. World Health Organization. Health Policy and Systems Research Agenda for Rehabilitation: Concept Note; World Health Organization: Geneva, Switzerland, 2019.

14. Jesus, T.S.; Hoenig, H. Crossing the global quality chasm: Where does rehabilitation stand? Arch. Phys. Med. Rehabil. 2019, 100, 2215-2217. [CrossRef] [PubMed]

15. Ghaffar, A.; Tran, N.; Langlois, E.; Shroff, Z.; Javadi, D. Alliance for Health Policy and Systems Research: Aims, achievements and ambitions. Public Health Res. Pract. 2017, 27, 2711703. [CrossRef]

16. Mills, A. Health policy and systems research: Defining the terrain; identifying the methods. Health Policy Plan. 2012, 27, 1-7. [CrossRef] [PubMed]

17. World Health Organization \& Alliance for Health Policy and Systems Research. Invest in Knowledge for Resilient Health Systems; World Health Organization \& Alliance for Health Policy and Systems Research: Geneva, Switzerland, 2016.

18. Peters, D.H. Health policy and systems research: The future of the field. Health Res. Policy Syst. 2018, 16, 84. [CrossRef]

19. Bennett, S.; Frenk, J.; Mills, A. The evolution of the field of Health Policy and Systems Research and outstanding challenges. Health Res. Policy Syst. 2018, 16, 43. [CrossRef] 
20. Sheikh, K.; Gilson, L.; Agyepong, I.A.; Hanson, K.; Ssengooba, F.; Bennett, S. Building the field of health policy and systems research: Framing the questions. PLoS Med. 2011, 8, e1001073. [CrossRef]

21. Adam, T.; Ahmad, S.; Bigdeli, M.; Ghaffar, A.; Rottingen, J.A. Trends in health policy and systems research over the past decade: Still too little capacity in low-income countries. PLoS ONE 2011, 6, e27263. [CrossRef]

22. Javadi, D.; Tran, N.; Ghaffar, A. Building a Workforce for Future Health Systems: Reflections from Health Policy and Systems Research. Health Serv. Res. 2018, 53, 4024-4033. [CrossRef]

23. Lohr, K.N.; Steinwachs, D.M. Health services research: An evolving definition of the field. Health Serv. Res. 2002, 37, 7-9.

24. Agency for Healthcare Research and Quality. Health Services Research: A Definition. Available online: https://www.ahrq.gov/funding/training-grants/hsrguide/hsrguide.html (accessed on 11 October 2019).

25. Bettger, J.P.; Nguyen, V.Q.; Thomas, J.G.; Guerrier, T.; Yang, Q.; Hirsch, M.A.; Pugh, T.; Harris, G.; Eller, M.A.; Pereira, C.; et al. Turning Data into Information: Opportunities to Advance Rehabilitation Quality, Research, and Policy. Arch. Phys. Med. Rehabil. 2018, 99, 1226-1231. [CrossRef] [PubMed]

26. Spyra, K.; Muller-Fahrnow, W. Rehabilitation as a subject of health services research. Bundesgesundheitsblatt Gesundheitsforschung Gesundheitsschutz 2006, 49, 188-197. [CrossRef] [PubMed]

27. Jette, A.M. Health Services Research in the 21st Century. Phys. Ther. 2019, 99, 255-257. [CrossRef] [PubMed]

28. Skempes, D.; Melvin, J.; von Groote, P.; Stucki, G.; Bickenbach, J. Using concept mapping to develop a human rights based indicator framework to assess country efforts to strengthen rehabilitation provision and policy: The Rehabilitation System Diagnosis and Dialogue framework (RESYST). Glob. Health 2018, 14, 96. [CrossRef] [PubMed]

29. Jesus, T.S.; Landry, M.D.; Dussault, G.; Fronteira, I. Human resources for health (and rehabilitation): Six Rehab-Workforce Challenges for the century. Hum. Resour. Health 2017, 15, 8. [CrossRef]

30. World Health Organization. Rehabilitation in Health Systems: Guide for Action; World Health Organization: Geneva, Switzerland, 2019.

31. Global Rehabilitation Alliance. About Us. Available online: http://global-rehabilitation-alliance.org/aboutus-1.html (accessed on 4 October 2019).

32. America Congress of Rehabilitation Medicine. Health Services Research Networking Group. Available online: https://acrm.org/acrm-communities/health-services-research/ (accessed on 5 October 2019).

33. National Institute on Disability, Independent Living, and Rehabilitation Research (NIDILRR). 2018-2023 Long-Range Plan 2019. Available online: https://acl.gov/sites/default/files/about-acl/2019-01/NIDILRR\% 20LRP-2018-2023-Final.pdf (accessed on 20 September 2019).

34. Resnik, L.; Freburger, J.K. Health Services Research: Physical Therapy Has Arrived! Phys. Ther. 2015, 95, $1605-1607$. [CrossRef]

35. Jesus, T.S. Systematic Reviews and Clinical Trials in Rehabilitation: Comprehensive Analyses of Publication Trends. Arch. Phys. Med. Rehabil. 2016, 97, 1853-1862. [CrossRef]

36. Jesus, T.S.; Colquhoun, H.L. Publication trends of study protocols in rehabilitation. Eur. J. Phys. Rehabil. Med. 2018, 54, 785-791. [CrossRef]

37. Jesus, T.S.G.S.; Castellini, G.; Colquhoun, H.; Brooks, D. Evolving physiotherapy research publications between 2005 and 2015. Physiothe. Can. 2019. [CrossRef]

38. Kamper, S.J.; Moseley, A.M.; Herbert, R.D.; Maher, C.G.; Elkins, M.R.; Sherrington, C. 15 years of tracking physiotherapy evidence on PEDro, where are we now? Br. J. Sports Med. 2015, 49, 907-909. [CrossRef]

39. Negrini, S.; Levack, W.; Gimigliano, F.; Arienti, C.; Villafane, J.H.; Kiekens, C. The Struggle for Evidence in Physical and Rehabilitation Medicine: Publication Rate of Randomized Controlled Trials and Systematic Reviews Is Growing More Than in Other Therapeutic Fields. Am. J. Phys. Med. Rehabil. 2019, 98, 258-265. [CrossRef] [PubMed]

40. English, K.M.; Pourbohloul, B. Increasing health policy and systems research capacity in low- and middle-income countries: Results from a bibliometric analysis. Health Res. Policy Syst. 2017, $15,64$. [CrossRef] [PubMed]

41. Macarayan, E.K.; Balabanova, D.; Gotsadze, G. Assessing the field of health policy and systems research using symposium abstract submissions and machine learning techniques. Health Policy Plan. 2019, 34, 721-731. [CrossRef] [PubMed]

42. English, K.M.; Pourbohloul, B. Health policy and systems research collaboration pathways: Lessons from a network science analysis. Health Res. Policy Syst. 2017, 15, 71. [CrossRef] 
43. Bouchard, L.; Albertini, M.; Batista, R.; de Montigny, J. Research on health inequalities: A bibliometric analysis (1966-2014). Soc. Sci. Med. 1982 2015, 141, 100-108. [CrossRef]

44. Defor, S.; Kwamie, A.; Agyepong, I.A. Understanding the state of health policy and systems research in West Africa and capacity strengthening needs: Scoping of peer-reviewed publications trends and patterns 1990-2015. Health Res. Policy Syst. 2017, 15, 55. [CrossRef]

45. Simpson, L.A.; Koechlein, L.; Menachemi, N.; Wolfe, M.J. Show Me the Money! Trends in Funding for Health Services Research. Health Serv. Res. 2018, 53, 3967-3975. [CrossRef]

46. Halladay, C.W.; Trikalinos, T.A.; Schmid, I.T.; Schmid, C.H.; Dahabreh, I.J. Using data sources beyond PubMed has a modest impact on the results of systematic reviews of therapeutic interventions. J. Clin. Epidemiol. 2015, 68, 1076-1084. [CrossRef]

47. Booth, A. Over 85\% of included studies in systematic reviews are on MEDLINE. J. Clin. Epidemiol. 2016, 79, 165-166. [CrossRef]

48. Mimouni, M.; Cismariu-Potash, K.; Ratmansky, M.; Shaklai, S.; Amir, H.; Mimouni-Bloch, A. Trends in Physical Medicine and Rehabilitation Publications Over the Past 16 Years. Arch. Phys. Med. Rehabil. 2016, 97, 1030-1033. [CrossRef] [PubMed]

49. Bastian, H.; Glasziou, P.; Chalmers, I. Seventy-five trials and eleven systematic reviews a day: How will we ever keep up? PLoS Med. 2010, 7, e1000326. [CrossRef] [PubMed]

50. Ma, Y.; Dong, M.; Zhou, K.; Mita, C.; Liu, J.; Wayne, P.M. Publication Trends in Acupuncture Research: A 20-Year Bibliometric Analysis Based on PubMed. PLoS ONE 2016, 11, e0168123. [CrossRef] [PubMed]

51. Hung, B.T.; Long, N.P.; Le Phi Hung, N.T.; Anh, N.H.; Nghi, T.D.; Van Hieu, M.; Trang, N.T.; Rafidinarivo, H.F.; Anh, N.K.; Hawkes, D.; et al. Research trends in evidence-based medicine: A joinpoint regression analysis of more than 50 years of publication data. PLOS ONE 2015, 10, e0121054. [CrossRef] [PubMed]

52. Richter, R.R.; Austin, T.M. Using MeSH (medical subject headings) to enhance PubMed search strategies for evidence-based practice in physical therapy. Phys. Ther. 2012, 92, 124-132. [CrossRef] [PubMed]

53. Jesus, T.S.; Bright, F.A.; Pinho, C.S.; Papadimitriou, C.; Kayes, N.M.; Cott, C.A. Scoping review of the person-centered literature in adult physical rehabilitation. Disabil. Rehabil. 2019, 1-11. [CrossRef]

54. Jesus, T.S.; Hoenig, H. Postacute rehabilitation quality of care: Toward a shared conceptual framework. Arch. Phys. Med. Rehabil. 2015, 96, 960-969. [CrossRef]

55. Meyer, T.; Gutenbrunner, C.; Bickenbach, J.; Cieza, A.; Melvin, J.; Stucki, G. Towards a conceptual description of rehabilitation as a health strategy. J. Rehabil. Med. 2011, 43, 765-769. [CrossRef]

56. European Physical and Rehabilitation Medicine Bodies Alliance. White Book on Physical and Rehabilitation Medicine (PRM) in Europe. Chapter 1. Definitions and concepts of PRM. Eur. J. Phys. Rehabil. Med. 2018, 54, 156-165.

57. O'Mara, A.; Rowland, J.H.; Greenwell, T.N.; Wiggs, C.L.; Fleg, J.; Joseph, L.; McGowan, J.; Panagis, J.S.; Washabaugh, C.; Peng, G.C.; et al. National Institutes of Health Research Plan on Rehabilitation: NIH Medical Rehabilitation Coordinating Committee. Phys. Ther. 2017, 97, 104-407.

58. MacLachlan, M.; McVeigh, J.; Cooke, M.; Ferri, D.; Holloway, C.; Austin, V.; Javadi, D. Intersections Between Systems Thinking and Market Shaping for Assistive Technology: The SMART (Systems-Market for Assistive and Related Technologies) Thinking Matrix. Int. J. Environ. Res. Public Health 2018, 15, 2627. [CrossRef] [PubMed]

59. Atkins, D.; Kilbourne, A.M.; Shulkin, D. Moving from Discovery to System-Wide Change: The Role of Research in a Learning Health Care System: Experience from Three Decades of Health Systems Research in the Veterans Health Administration. Annu. Rev. Public Health 2017, 38, 467-487. [CrossRef] [PubMed]

60. Institute of Medicine. Best Care at Lower Cost: The Path to Continuously Learning Health Care in America; Institute of Medicine: Washington, DC, USA, 2012.

61. Jesus, T.S.; Landry, M.; Brooks, D.; Hoenig, H. Physical Rehabilitation Needs Per Condition Type: Results from the Global Burden of Disease study 2017. Arch. Phys. Med. Rehabi. 2019.

62. Louw, Q.; Twizeyemariya, A.; Grimmer, K.; Leibbrandt, D. Estimating the costs and benefits of stroke rehabilitation in South Africa. J. Eval. Clin. Pract. 2019. [CrossRef]

63. Howard-Wilsher, S.; Irvine, L.; Fan, H.; Shakespeare, T.; Suhrcke, M.; Horton, S.; Poland, F.; Hooper, L.; Song, F. Systematic overview of economic evaluations of health-related rehabilitation. Disabil. Health J. 2016, 9, 11-25. [CrossRef]

64. Shields, G.E.; Wells, A.; Doherty, P.; Heagerty, A.; Buck, D.; Davies, L.M. Cost-effectiveness of cardiac rehabilitation: A systematic review. Heart Br. Card. Soc. 2018, 104, 1403-1410. [CrossRef] 
65. Burge, E.; Monnin, D.; Berchtold, A.; Allet, L. Cost-Effectiveness of Physical Therapy Only and of Usual Care for Various Health Conditions: Systematic Review. Phys. Ther. 2016, 96, 774-786. [CrossRef]

66. World Health Organization \& the World Bank. World Report on Disability; World Health Organization \& the World Bank: Geneva, Switzerland, 2011.

67. Menachemi, N.; Simpson, L.A.; Wolfe, M.J. Positioning the Health Services Research Workforce for Continued Success: Recommendations from AcademyHealth Stakeholders. Health Serv. Res. 2018, 53, 4041-4050. [CrossRef]

68. Mor, V.; Wallace, P. Funding the Training of Future Health Services Researchers. Health Serv. Res. 2018, 53, $3976-3984$. [CrossRef]

69. Frogner, B.K. Update on the Stock and Supply of Health Services Researchers in the United States. Health Serv. Res. 2018, 53, 3945-3966. [CrossRef]

70. Lembani, M.; Teddy, G.; Molosiwa, D.; Hwabamungu, B. Post-doctoral research fellowship as a health policy and systems research capacity development intervention: A case of the CHESAI initiative. Health Res. Policy Syst. 2016, 14, 89. [CrossRef] [PubMed]

71. Izugbara, C.O.; Kabiru, C.W.; Amendah, D.; Dimbuene, Z.T.; Donfouet, H.P.; Atake, E.H.; Ingabire, M.G.; Maluka, S.; Mumah, J.N.; Mwau, M.; et al. "It takes more than a fellowship program”: Reflections on capacity strengthening for health systems research in sub-Saharan Africa. BMC Health Serv. Res. 2017, 17, 696. [CrossRef] [PubMed]

72. Bowsher, G.; Papamichail, A.; El Achi, N.; Ekzayez, A.; Roberts, B.; Sullivan, R.; Patel, P. A narrative review of health research capacity strengthening in low and middle-income countries: Lessons for conflict-affected areas. Glob. Health 2019, 15, 23. [CrossRef] [PubMed]

73. Moses, H.; Matheson, D.H., III; Cairns-Smith, S.; George, B.P.; Palisch, C.; Dorsey, E.R. The anatomy of medical research: US and international comparisons. JAMA 2015, 313, 174-189. [CrossRef] [PubMed]

74. Nugraha, B.; Setyono, G.R.; Defi, I.R.; Gutenbrunner, C. Strengthening rehabilitation services in Indonesia: A brief situation analysis. J. Rehabil. Med. 2018, 50, 377-384. [CrossRef] [PubMed]

75. Gutenbrunner, C.; Nugraha, B. Responding to the World Health Organization Global Disability Action Plan in Egypt: A Technical Consultancy to develop a National Disability, Health and Rehabilitation Plan. J. Rehabil. Med. 2018, 50, 333-337. [CrossRef] 